\title{
The Integrated Density of States for an Interacting Multiparticle Homogeneous Model and Applications to the Anderson Model
}

\author{
Frédéric Klopp ${ }^{1,2}$ and Heribert Zenk ${ }^{3}$ \\ ${ }^{1}$ LAGA, Institut Galilée, Université Paris-Nord, 93430 Villetaneuse, France \\ ${ }^{2}$ Institut Universitaire de France, 75005 Paris, France \\ ${ }^{3}$ Mathematisches Institut, Ludwig-Maximilians-Universität, Theresienstraße 39, \\ 80333 München, Germany
}

Correspondence should be addressed to Heribert Zenk, zenk@mathematik.uni-muenchen.de

Received 8 July 2008; Revised 9 October 2008; Accepted 13 January 2009

Recommended by Valentin Zagrebnov

For a system of $n$ interacting particles moving in the background of a "homogeneous" potential, we show that if the single particle Hamiltonian admits a density of states, so does the interacting $n$-particle Hamiltonian. Moreover, this integrated density of states coincides with that of the free particle Hamiltonian. For the interacting $n$-particle Anderson model, we prove regularity properties of the integrated density of states by establishing a Wegner estimate.

Copyright (C) 2009 F. Klopp and H. Zenk. This is an open access article distributed under the Creative Commons Attribution License, which permits unrestricted use, distribution, and reproduction in any medium, provided the original work is properly cited.

\section{Introduction}

Recently, models describing interacting quantum particles in a random potential have been studied (see, e.g., [1-3]). We consider $n$ interacting particles moving in a "homogeneous" potential in the $d$-dimensional configuration space $\mathbb{R}^{d}$. A typical example of what we mean by a "homogeneous" potential is an Anderson or alloy-type random potential. The goal of the present paper is twofold.

First, we prove that if the Hamiltonian of the single particle in the "homogeneous" media admits an integrated density of states (IDS), then, so does the interacting $n$-particle Hamiltonian. The proof consists of two steps. First, we prove the claim for the noninteracting $n$-particle system and in a second step, we show that the IDS for noninteracting and interacting system is the same. These two steps allow an application to the interacting $n$ particle Anderson model in $\mathbb{R}^{d}$.

Note that, in general, knowledge of the integrated density of states is not yielding estimates for the normalized counting functions of the finite volume restrictions of the random operator; such information is also very valuable as it is a major tool in the study 
of the spectrum. Therefore, the second aim of this note is to provide estimates on the finite volume normalized counting function which lead to a Wegner estimate. The proof uses the ideology and tools developed for the one-particle Hamiltonian.

\subsection{The Interacting Multiparticle Model}

The noninteracting $n$-particle Hamiltonian satisfies $H_{0}^{n}=-\Delta+V_{\text {ext }}^{n}$ where the Laplacian $-\Delta$ on $\mathbb{R}^{n d}$ describes the free kinetic energy of the $n$ particles. As all the particles are in the same background, the potential $V_{\mathrm{ext}}^{n}$ is of the form

$$
V_{\mathrm{ext}}^{n}\left(x_{1}, \ldots, x_{n}\right)=\sum_{k=1}^{n} V^{1}\left(x_{k}\right)
$$

Hence, the noninteracting $n$-particle Hamiltonian is a sum of one-particle Hamiltonians $H^{1}=$ $-\Delta+V^{1}$. On the one particle potential $V^{1}$, we assume that

(H.1.a) $\left(V^{1}\right)_{+}:=\max \left\{V^{1}, 0\right\}$ is locally square integrable and $\left(V^{1}\right)_{-}:=\max \left\{-V^{1}, 0\right\}$ is an infinitesimally $-\Delta$-bounded potential, that is, $\Phi\left(\left(V^{1}\right)_{-}\right) \supseteq \Phi(-\Delta)$ and for all $\alpha>0$, there exists $\gamma(\alpha)<\infty$, such that for all $\phi \in \Phi(-\Delta)$

$$
\left\|\left(V^{1}\right)_{-} \phi\right\| \leq \alpha\|\Delta \phi\|+\gamma(\alpha)\|\phi\|,
$$

(H.1.b) the operator $H^{1}$ admits an integrated density of states, say $N_{1}$, that is, if $H_{0 . L}^{1}$ denotes the Dirichlet restriction of $H^{1}$ to a cube $\Lambda(0, L)$ centered at 0 of side-length, $L$, then the following limit exists

$$
N_{1}(E):=\lim _{L \rightarrow+\infty} L^{-d} \operatorname{Trace}\left(\mathbf{1}_{]-\infty, E]}\left(H_{0 . L}^{1}\right)\right) .
$$

Assumption (H.1.a) implies essential self-adjointness of $-\Delta+V^{1}$ on $C_{0}^{\infty}\left(\mathbb{R}^{d}\right)$ by $[4$, Theorem X.29 ]. Indeed,

$$
V_{\mathrm{ext}}^{n}=\left(V_{\mathrm{ext}}^{n}\right)_{+}-\left(V_{\mathrm{ext}}^{n}\right)_{-^{\prime}} \quad\left(V_{\mathrm{ext}}^{n}\right)_{ \pm}\left(x_{1}, \ldots, x_{n}\right):=\sum_{j=1}^{n}\left(V^{1}\right)_{ \pm}\left(x_{j}\right),
$$

where

(i) $\left(V_{\text {ext }}^{n}\right)_{-}$is infinitesimally $-\Delta$-bounded, that is, (1.2) holds for the same constants and the Laplacian over $\mathbb{R}^{n d}$;

(ii) $\left(V_{\mathrm{ext}}^{n}\right)_{+}$is nonnegative locally square integrable.

The self-adjoint extensions of $-\Delta+V^{1}$ and $-\Delta+V_{\mathrm{ext}}^{n}$ are again denoted by $H^{1}$ and $H_{0}^{n}$; they are bounded from what follows.

Classical models for which the IDS is known to exist include periodic, quasiperiodic, and ergodic random Schrödinger operators (see, e.g., [5]). 
In the definition of the density of states, we could also have considered the case of Neumann or other boundary conditions.

The interacting $n$-particle Hamiltonian is of the form

$$
H^{n}:=-\Delta+V_{i}^{n}+V_{\mathrm{ext}}^{n}
$$

where

$$
V_{i}^{n}\left(x_{1}, \ldots, x_{n}\right):=\sum_{1 \leq k<l \leq n} V\left(x_{k}-x_{l}\right)
$$

is a localized repulsive interaction potential generated by the particles; so we assume that

(H.2) $V: \mathbb{R}^{d} \rightarrow \mathbb{R}$ is measurable nonnegative locally square integrable and $V$ tends to 0 at infinity.

The standard repulsive interaction in three-dimensional space is of course the Coulomb interaction $V(x)=1 /|x|$. In some cases, due to screening, it must be replaced by the Yukawa's interaction $V(x)=e^{-|x|} /|x|$.

Finally, we make one more assumption on both $V^{1}$ and $V$; we assume that

(H.3) the operator $V_{i}^{n}\left(H_{0}^{n}-i\right)^{-1}$ is bounded.

Assumption (H.3) is satisfied in the case of the Coulomb and Yukawa potential for those $V^{1}$ satisfying (H.1.a); $H_{0}^{n}$ is self-adjoint on $\mathscr{\Phi}\left(H_{0}^{n}\right) \subseteq \Phi(-\Delta)$, hence $\left\|V_{i}^{n}\left(H_{0}^{n}-i\right)^{-1}\right\| \leq$ $\left\|V_{i}^{n}(-\Delta-i)^{-1}\right\| \cdot\left\|(-\Delta-i)\left(H_{0}^{n}-i\right)^{-1}\right\|$, where $\left\|(-\Delta-i)\left(H_{0}^{n}-i\right)^{-1}\right\|<\infty$ due to closed graph theorem and $\left\|V_{i}^{n}(-\Delta-i)^{-1}\right\|<\infty$ for Coulomb and Yukawa's interaction potentials $V_{i}^{n}$; see $[4$, Theorem X.16 ].

\section{The Integrated Density of States}

We now compute the IDS for the $n$-particle model. Let $\Lambda_{L}=\Lambda(0, L)$ be the cube in $\mathbb{R}^{d}$ centered at 0 with side-length $L$ and write $\Lambda_{L}^{n}=\Lambda_{L} \times \cdots \times \Lambda_{L}$ for the product of $n$ copies of $\Lambda_{L}$. We denote the restriction of the interacting $n$-particle Hamiltonian $H^{n}$ to $\Lambda_{L}^{n}$ with Dirichlet boundary conditions by $H_{L}^{n}$. Clearly assumptions (H.2) and (H.1.a) guarantee that $H_{L}^{n}$ is bounded from what follows with compact resolvent. Hence, for any $E \in \mathbb{R}$, one defines the normalized counting functions

$$
N_{L}(E):=L^{-n d} \operatorname{Trace}\left(\mathbf{1}_{-\infty, E]}\left(H_{L}^{n}\right)\right) .
$$

As usual, $N$, the IDS of $H^{n}$ is defined as the limit of $N_{L}(E)$ when $L \rightarrow+\infty$. Equivalently, one can define the density of states measure applied to a test function $\varphi$ as the limit of $L^{-n d}$ Trace $\left[\varphi\left(H_{L}^{n}\right)\right]$. If the limit exists, it defines a nonnegative measure. It is a classical result that the existence of that limit (for all test functions) or that of $N_{L}(E)$ is equivalent [5]. 


\subsection{The IDS for the Noninteracting $n$-Particle System}

Recall that, by assumption (H.1.b), the single particle model $H^{1}$ admits an IDS (see [5]) and a density of states measure denoted, respectively, by $N_{1}$ and $v_{1}$.

Let $H_{0, L}^{n}$ be the restriction of $H_{0}^{n}$ to $\Lambda_{L}^{n}$ with Dirichlet boundary conditions. One has the following lemma.

Lemma 2.1. The IDS for the noninteracting n-particle Boltzmann model given by

$$
N_{n i}(E):=\lim _{L \rightarrow \infty} \frac{1}{L^{n d}} \operatorname{Trace}\left(\mathbf{1}_{]-\infty, E]}\left(H_{0, L}^{n}\right)\right)
$$

exists and satisfies

$$
N_{n i}=N_{1} * v_{1} * \cdots * v_{1} .
$$

Let us comment on this result. First, the convolution product in (2.3) makes sense as all the measures and functions are supported on half-axes of the form $[a,+\infty)$; this results from assumption (H.1.a). When the field $V^{1}$ is not bounded from what follows, one will need some estimate on the decay of $N_{1}$ and $v_{1}$ near $-\infty$ to make sense of (2.3) (and to prove it); such estimates are known for some models (see, e.g., [5, 6]).

Proof of Lemma 2.1. The operator $H_{0}^{n}$ is the sum of $n$ commuting Hamiltonians, each of which is unitarily equivalent to $H^{1}$; so is $H_{0, L}^{n}$, its restriction to the cube $\Lambda_{L}^{n}$. As the sum decomposition of $H_{0}^{n}$ commutes with the restriction to $\Lambda_{L}^{n}$, the eigenvalues of $H_{0, L}^{n}$ are exactly the sum of $n$ eigenvalues of $H^{1}$ restricted to $\Lambda_{L}$. This immediately yields that

$$
\operatorname{Trace}\left(\mathbf{1}_{]-\infty, E]}\left(H_{0, L}^{n}\right)\right)=\left(\widehat{N}_{1}^{L} * \widehat{v}_{1}^{L} * \cdots * \widehat{v}_{1}^{L}\right)(E),
$$

where $\widehat{N}_{1}^{L}(E)$ is the eigenvalue counting function for $H^{1}$ restricted to $\Lambda_{L}$, and $\widehat{v}_{1}^{L}$ is its counting measure (i.e., $\left.d \widehat{N}_{1}^{L}\right)$. The normalized counting function and measure, $N_{1}^{L}$ and $v_{1}^{L}$, are defined as

$$
N_{1}^{L}=\frac{1}{L^{d}} \widehat{N}_{1}^{L}, \quad v_{1}^{L}=\frac{1}{L^{d}} \widehat{v}_{1}^{L} .
$$

The existence of the density of states of $H^{1}$ then exactly says that $N_{1}^{L}$ and $v_{1}^{L}$ converge, respectively, to $N_{1}$ and $v_{1}$. The convergence of $N_{1}^{L} * \nu_{1}^{L} * \cdots * v_{1}^{L}$ to $N_{1} * v_{1} * \cdots * v_{1}$ is then guaranteed as the convolution is bilinear bicontinuous operation on distributions. This completes the proof of Lemma 2.1.

Let us now say a word on the boundary conditions chosen to define the IDS. Here, we chose to define it as an infinite-volume limit of the normalized counting for Dirichlet eigenvalues. Clearly, if we know that the single particle Hamiltonian has an IDS defined as the infinite-volume limit of the normalized counting for Neumann eigenvalues, so does the noninteracting $n$-body Hamiltonian. Moreover, in the case when the two limits coincide for the one-body Hamiltonian, they also coincide for the noninteracting $n$-body Hamiltonian. 
Using Dirichlet-Neumann bracketing, one then sees that the integrated densities of states for both the one-body and noninteracting $n$-body Hamiltonian for positive mixed boundary conditions also exist and coincide with that defined with either Dirichlet or Neumann boundary conditions.

\subsection{Existence of the IDS for the Interacting n-Particle System}

Let $H_{L}^{n}$ denote the restriction of $H^{n}$ to the box $\Lambda_{L}^{n}$ with Dirichlet boundary conditions. Our main result is.

Theorem 2.2. Assume (H.1), (H.2), and (H.3) are satisfied. For any $\varphi \in C_{0}^{\infty}(\mathbb{R})$, one has

$$
\frac{1}{L^{n d}} \operatorname{Trace}\left[\varphi\left(H_{L}^{n}\right)-\varphi\left(H_{0, L}^{n}\right)\right] \stackrel{L \rightarrow \infty}{\longrightarrow} 0 .
$$

As the density of states measure of $H^{n}$ is defined by

$$
\langle\varphi, d N\rangle=\lim _{L \rightarrow+\infty} \frac{1}{L^{n d}} \text { Trace }\left[\varphi\left(H_{L}^{n}\right)\right]
$$

we immediately get the following corollary.

Corollary 2.3. Assume (H.1), (H.2), and (H.3) are satisfied. The IDS for the interacting n-particle Boltzmann model $H^{n}$ exists and coincides with that of the noninteracting model $H_{0}^{n}$; hence, it satisfies

$$
N=N_{n i}=N_{1} * v_{1} * \cdots * v_{1}
$$

Note that, in view of the remark concluding Section 2.1, we see that the integrated density of states of the interacting $n$-body Hamiltonian is independent of the boundary conditions if that of the one-body Hamiltonian is.

In Corollary 2.3, we dealt with the Boltzmann statistic, that is, without statistic. Theorem 2.2 stays clearly true for both the Fermi and the Bose statistics, that is, if one restricts to the subspaces of symmetric and antisymmetric functions. One defines the following:

(i) for the Fermi statistics, the Fermi integrated density of states

$$
\left\langle\varphi, d N^{F}\right\rangle=\lim _{L \rightarrow+\infty} \frac{n !}{L^{n d}} \operatorname{Trace}_{\wedge_{n} L^{2}\left(\Lambda_{L}^{1}\right)}\left[\varphi\left(H_{L}^{n}\right)\right]
$$

where $\wedge_{n} L^{2}\left(\Lambda_{L}^{1}\right)$ denotes $n$-fold antisymmetric tensor product of $L^{2}\left(\Lambda_{L}^{1}\right)$;

(ii) for the Bose statistics, the Bose integrated density of states

$$
\left\langle\varphi, d N^{B}\right\rangle=\lim _{L \rightarrow+\infty} \frac{n !}{L^{n d}} \operatorname{Trace}_{\oplus_{n} L^{2}\left(\Lambda_{L}^{1}\right)}\left[\varphi\left(H_{L}^{n}\right)\right],
$$

where $\stackrel{s}{\oplus_{n}} L^{2}\left(\Lambda_{L}^{1}\right)$ denotes $n$-fold symmetric tensor product of $L^{2}\left(\Lambda_{L}^{1}\right)$. 
Let us now discuss shortly the Bose and Fermi counting functions (i.e., the eigenvalue counting functions of the Hamiltonian restricted to a finite cube) in the free case (i.e., when the interaction vanishes). Consider the cube $\Lambda_{L}^{1}$ and let $E_{1}(L) \leq E_{2}(L) \leq \cdots$ be the eigenvalue of the single particle Hamiltonian repeated according to multiplicity. The three counting functions are then given by

$$
\begin{aligned}
\#_{L}(E) & :=\#\left\{\text { eigenvalues of } H_{0, L}^{n} \text { on } L^{2}\left(\Lambda_{L}^{n}\right) \text { less than } E\right\} \\
& =\#\left\{\left(j_{1}, j_{2}, \ldots, j_{n}\right): E_{j_{1}}(L)+E_{j_{2}}(L)+\cdots+E_{j_{n}}(L) \leq E\right\}, \\
\#_{L}^{F}(E) & :=\#\left\{\text { eigenvalues of } H_{0, L}^{n} \text { on } \wedge_{n} L^{2}\left(\Lambda_{L}^{1}\right) \text { less than } E\right\} \\
& =\#\left\{\left(j_{1}, j_{2}, \ldots, j_{n}\right): j_{1}<j_{2}<\cdots<j_{n}, E_{j_{1}}(L)+\cdots+E_{j_{n}}(L) \leq E\right\}, \\
\#_{L}^{B}(E) & :=\#\left\{\text { eigenvalues of } H_{0, L}^{n} \text { on } \stackrel{s}{\oplus}_{n} L^{2}\left(\Lambda_{L}^{1}\right) \text { less than } E\right\} \\
& =\#\left\{\left(j_{1}, j_{2}, \ldots, j_{n}\right): j_{1} \leq j_{2} \leq \cdots \leq j_{n}, E_{j_{1}}(L)+\cdots+E_{j_{n}}(L) \leq E\right\} .
\end{aligned}
$$

Hence,

$$
n ! \#_{L}^{F}(E) \leq \#_{L}(E) \leq n ! \#_{L}^{B}(E)
$$

Uniformly in $L$, the eigenvalues $\left(E_{j}(L)\right)_{j \geq 1}$ are lower bounded by, say, $-C$. Hence, if $E_{j_{1}}(L)+$ $E_{j_{2}}(L)+\cdots+E_{j_{n}}(L) \leq E$, then, for $k=1, \ldots, n$, one has $E_{j_{k}}(L) \leq E+C n$ so that $j_{k} \leq \widehat{N}_{1}^{L}(E+C n)=$ $N_{1}^{L}(E+C n) L^{d}$. This implies that

$$
\begin{aligned}
0 & \leq \#_{L}^{B}(E)-\#_{L}^{F}(E) \\
& =\#\left\{\left(j_{1}, j_{2}, \ldots, j_{n}\right) ; \quad \begin{array}{c}
j_{1} \leq j_{2} \leq \cdots \leq j_{n}, \quad \exists k<l \text { s.t. } j_{k}=j_{l} \\
E_{j_{1}}(L)+E_{j_{2}}(L)+\cdots+E_{j_{n}}(L) \leq E
\end{array}\right\} \\
& \leq \widetilde{C} L^{d(n-1)} .
\end{aligned}
$$

Thus, dividing (2.12) and (2.13) by $L^{\text {nd }}$ and taking the limit $L \rightarrow+\infty$, we obtain that the free Fermi and Bose density of states are equal to the Boltzmann one. Theorem 2.2 then gives the following corollary.

Corollary 2.4. Assume (H.1), (H.2), and (H.3) are satisfied. One has $N=N^{B}=N^{F}$.

Proof of Theorem 2.2. We take some $q>n d / 2$ and specify the appropriate choice later on. By assumptions (H.1.a) and (H.2), there exists $\zeta>0$ such that

$$
-\infty<-\zeta \leq \min \left(\inf _{L \geq 1}\left\{\inf \left[\sigma\left(H_{0, L}^{n}\right) \cup \sigma\left(H_{L}^{n}\right)\right]\right\}, \inf \left[\sigma\left(H_{0}^{n}\right) \cup \sigma\left(H^{n}\right)\right]\right) .
$$

Let $\gamma=\gamma(1 / 2)$ be given by (1.2) for $\alpha=1 / 2$. Fix $\lambda_{0}>\zeta+2 \gamma+1$. 
By (2.14), we only need to prove (2.6) for $\varphi \in C_{0}^{\infty}(\mathbb{R})$ supported in $(-\zeta-1,+\infty)$. For such a function, let $\tilde{\varphi}$ be an almost analytic extension of the function $x \mapsto\left(x+\lambda_{0}\right)^{q} \varphi(x) \in C_{0}^{\infty}(\mathbb{R})$, that is, $\tilde{\varphi}$ satisfies

(i) $\tilde{\varphi} \in \mathcal{S}(\{z \in \mathbb{C}:|\Im z|<1\}$,

(ii) for any $k \in \mathbb{N}$, the family of functions $\left(x \mapsto(\partial \tilde{\varphi} / \partial \bar{z})(x+i y)|y|^{-k}\right)_{0<|y|<1}$ is bounded in $\mathcal{S}(\mathbb{R})$.

The functional calculus based on the Helffer-Sjöstrand formula implies

$$
\varphi\left(H_{L}^{n}\right)-\varphi\left(H_{0, L}^{n}\right)=\frac{i}{2 \pi} \int_{\mathbb{C}} \frac{\partial \tilde{\varphi}}{\partial \bar{z}}(z)\left[\left(H_{L}^{n}+\lambda_{0}\right)^{-q}\left(H_{L}^{n}-z\right)^{-1}-\left(H_{0, L}^{n}+\lambda_{0}\right)^{-q}\left(H_{0, L}^{n}-z\right)^{-1}\right] d z \wedge d \bar{z}
$$

In the following, we apply an idea, which has already been used in [6,7] and which simplifies in this situation. Using resolvent equality, the integrand in (2.15) is written as

$$
\begin{aligned}
\left(H_{L}^{n}+\lambda_{0}\right)^{-q}\left(H_{L}^{n}-z\right)^{-1}-\left(H_{0, L}^{n}+\lambda_{0}\right)^{-q}\left(H_{0, L}^{n}-z\right)^{-1} \\
=\left(H_{0, L}^{n}+\lambda_{0}\right)^{-q}\left[\left(H_{L}^{n}-z\right)^{-1}-\left(H_{0, L}^{n}-z\right)^{-1}\right] \\
+\left[\left(H_{L}^{n}+\lambda_{0}\right)^{-q}-\left(H_{0, L}^{n}+\lambda_{0}\right)^{-q}\right]\left(H_{L}^{n}-z\right)^{-1} \\
=-\left(H_{0, L}^{n}+\lambda_{0}\right)^{-q}\left(H_{0, L}^{n}-z\right)^{-1}\left(V_{i}^{n}\right)\left(H_{L}^{n}-z\right)^{-1} \\
-\sum_{l=1}^{q}\left(H_{0, L}^{n}+\lambda_{0}\right)^{l-q-1}\left(V_{i}^{n}\right)\left(H_{L}^{n}+\lambda_{0}\right)^{-l}\left(H_{L}^{n}-z\right)^{-1} .
\end{aligned}
$$

Estimating the trace of (2.16), we choose $\varepsilon>0$ and write

$$
V_{i}^{n}=V_{i}^{n} \cdot \mathbf{1}_{\left\{\left|V_{i}^{n}\right| \leq \varepsilon\right\}}+V_{i}^{n} \cdot \mathbf{1}_{\left\{\left|V_{i}^{n}\right|>\varepsilon\right\}}
$$

and note that $V_{i}^{n} \cdot \mathbf{1}_{\left\{\left|V_{i}^{n}\right| \leq \varepsilon\right\}}$ is bounded by $\left\|V_{i}^{n} \cdot \mathbf{1}_{\left\{\left|V_{i}^{n}\right| \leq \varepsilon\right\}}\right\| \leq \varepsilon$. As $V$ is nonnegative, one has

$$
\operatorname{supp}\left(V_{i}^{n} \cdot \mathbf{1}_{\left\{\left|V_{i}^{n}\right|>\varepsilon\right\}}\right) \subseteq \bigcup_{j=1}^{n} \bigcup_{\substack{i=1 \\ i \neq j}}^{n}\left\{\left(x_{1}, \ldots, x_{n}\right) \in \mathbb{R}^{n d}: V\left(x_{i}-x_{j}\right) \geq \frac{\varepsilon}{n(n-1)}\right\}
$$

As, by assumption (H.2), $V$ tends to 0 at infinity, (2.18) implies that there exists $0<C(n ; \varepsilon)$ (independent of $L$ ) such that

$$
\mu\left(\left\{\left|V_{i}^{n}\right|>\varepsilon\right\} \cap \Lambda_{L}^{n}\right) \leq C(n, \varepsilon) L^{(n-1) d},
$$


where $\mu(\cdot)$ denotes the Lebesgue measure. Using decomposition (2.17) of $V_{i}^{n}$, we obtain

$$
\begin{aligned}
\mid \operatorname{Trace} & \left(H_{0, L}^{n}+\lambda_{0}\right)^{-q}\left(H_{0, L}^{n}-z\right)^{-1}\left(V_{i}^{n}\right)\left(H_{L}^{n}-z\right)^{-1} \mid \\
\leq & \frac{\varepsilon}{|\Im z|^{2}} \operatorname{Trace}\left|\left(H_{0, L}^{n}+\lambda_{0}\right)^{-q}\right|+\frac{1}{|\Im z|}\left\|\left(V_{i}^{n}\right)\left(H_{L}^{n}-z\right)^{-1}\right\| \\
& \cdot \operatorname{Trace} \mid\left(H_{0, L}^{n}+\lambda_{0}\right)^{-q} \mathbf{1}_{\left\{\left|V_{i}^{n}\right|>\varepsilon\right\} \cap \Lambda_{L}^{n} \mid} \\
\leq & \frac{\varepsilon}{|\Im z|^{2}}\left\|\left(H_{0, L}^{n}+\lambda_{0}\right)^{-1}\right\|_{\tau_{q}}^{q}+\frac{1}{|\Im z|^{2}}\left\|\left(H_{0, L}^{n}+\lambda_{0}\right)^{-1}\right\|_{\tau_{q}}^{q-1} \\
& \cdot\left\|\left(H_{0, L}^{n}+\lambda_{0}\right)^{-1} \mathbf{1}_{\left\{\left|V_{i}^{n}\right|>\varepsilon\right\} \cap \Lambda_{L}^{n}}\right\|_{\tau_{q}} \cdot\left\|\left(V_{i}^{n}\right)\left(H_{0, L}^{n}+\lambda_{0}\right)^{-1}\right\|,
\end{aligned}
$$

where $\|\cdot\|_{\tau_{q}}$ denotes the $q$ th Schatten class norm (see [8]) and we used Hölder's inequality. In the same way, the cyclicity of the trace yields

$$
\begin{aligned}
& \left|\operatorname{Trace}\left(H_{0, L}^{n}+\lambda_{0}\right)^{l-q-1}\left(V_{i}^{n}\right)\left(H_{L}^{n}+\lambda_{0}\right)^{-l}\left(H_{L}^{n}-z\right)^{-1}\right| \\
& \leq \text { Trace }\left|\left(H_{L}^{n}+\lambda_{0}\right)^{-l}\left(H_{0, L}^{n}+\lambda_{0}\right)^{l-q-1}\left(V_{i}^{n}\right)\left(H_{L}^{n}-z\right)^{-1}\right| \\
& \leq\left\|\left(H_{L}^{n}+\lambda_{0}\right)^{-l}\left(H_{0, L}^{n}+\lambda_{0}\right)^{l}\right\| \cdot \operatorname{Trace}\left|\left(H_{0, L}^{n}+\lambda_{0}\right)^{-q-1}\left(V_{i}^{n}\right)\left(H_{L}^{n}-z\right)^{-1}\right| \\
& \leq \frac{C}{|\Im z|}\left\|\left(H_{0, L}^{n}+\lambda_{0}\right)^{-1}\right\|_{\tau_{q}}^{q-1} \cdot\left\|\left(H_{0, L}^{n}+\lambda_{0}\right)^{-1} \mathbf{1}_{\left\{|| V_{i}^{n} \mid>\varepsilon\right\} \cap \Lambda_{L}^{n}}\right\| \tau_{q} \cdot\left\|\left(V_{i}^{n}\right)\left(H_{0, L}^{n}+\lambda_{0}\right)^{-1}\right\| \\
& +C \frac{\varepsilon}{|\Im z|}\left\|\left(H_{0, L}^{n}+\lambda_{0}\right)^{-1}\right\|_{\tau_{q}}^{q} .
\end{aligned}
$$

We are now left with estimating $\left\|\left(H_{0, L}^{n}+\lambda_{0}\right)^{-1}\right\|_{\tau_{q}}$ and $\left\|\left(H_{0, L}^{n}+\lambda_{0}\right)^{-1} \mathbf{1}_{\left\{\left|V_{i}^{n}\right|>\varepsilon\right\} \cap \Lambda_{L}^{n}}\right\|_{\tau_{q}}$ for $q$ sufficiently large, depending on $n d$. Therefore, we compute

$$
\begin{aligned}
\left\|\left(H_{0, L}^{n}+\lambda_{0}\right)^{-1} \mathbf{1}_{\left\{\left|V_{i}^{n}\right|>\varepsilon\right\} \cap \Lambda_{L}^{n}}\right\|_{\tau_{q}} \leq & \left\|\left(H_{0, L}^{n}+\lambda_{0}\right)^{-1}\left(-\Delta_{\Lambda_{L}^{n}}+\lambda_{0}\right)^{1 / 2}\right\|_{\tau_{2 q}} \\
& \cdot\left\|\left(-\Delta_{\Lambda_{L}^{n}}+\lambda_{0}\right)^{-(1 / 2)} \mathbf{1}_{\left\{\left|V_{i}^{n}\right|>\varepsilon\right\} \cap \Lambda_{L}^{n}}\right\|_{\tau_{2 q}},
\end{aligned}
$$

where $-\Delta_{\Lambda_{L}^{n}}$ is the Dirichlet Laplacian on $\Lambda_{L}^{n}$. We use the decomposition (1.4). As the Laplacians are positive, the infinitesimal $-\Delta$-boundedness on $\left(V_{\mathrm{ext}}^{n}\right)_{-},[4$, Theorem X.18 ] and the definition of $\gamma$ imply the following form bound:

$$
\left|\left\langle\phi,\left(V_{\mathrm{ext}}^{n}\right)_{-} \phi\right\rangle\right| \leq \frac{1}{2}\left\langle\phi,-\Delta_{\Lambda_{L}^{n}} \phi\right\rangle+\gamma\|\phi\|^{2}
$$

As $\lambda_{0}>2 \gamma+1$, one has

$$
H_{0, L}^{n}+\lambda_{0} \geq-\Delta_{\Lambda_{L}^{n}}+\left(V_{\mathrm{ext}}^{n}\right)_{-}+\lambda_{0} \geq \frac{1}{2}\left(-\Delta_{\Lambda_{L}^{n}}-2 \gamma+2 \lambda_{0}\right) \geq \frac{1}{2}\left(-\Delta_{\Lambda_{L}^{n}}+\lambda_{0}\right) .
$$


Thus, the operator $H_{0, L}^{n}+\lambda_{0}$ is invertible and

$$
\left(H_{0, L}^{n}+\lambda_{0}\right)^{-1} \leq 2\left(-\Delta_{\Lambda_{L}^{n}}+\lambda_{0}\right)^{-1}
$$

Let $\left(\mu_{\underline{j}}\right)_{j}$ and $\left(\phi_{\underline{j}}\right)_{j}$, respectively, denote the eigenvalues and eigenfunctions of the Dirichlet Laplacian $-\Delta_{\Lambda_{L}^{n}}\left(\right.$ the index $j$ runs over $\left.\left(\mathbb{N}^{n d}\right)^{*}\right)$. For $q \in \mathbb{N}$ such that $2 q>n d$, we compute

$$
\begin{aligned}
\left\|\left(H_{0, L}^{n}+\lambda_{0}\right)^{-1}\left(-\Delta_{\Lambda_{L}^{n}}+\lambda_{0}\right)^{1 / 2}\right\|_{\tau_{2 q}}^{2 q} & =\sum_{\underline{j} \in \mathbb{N} n d}\left(\mu_{\underline{j}}\left(-\Delta_{\Lambda_{L}^{n}}\right)+\lambda_{0}\right)^{q}\left\langle\phi_{\underline{j}}\left(H_{0, L}^{n}+\lambda_{0}\right)^{-1} \phi_{\underline{j}}\right\rangle^{2 q} \\
& \left.\leq 2^{2 q} \sum_{\underline{j} \in \mathbb{N} n d}\left(\mu_{\underline{j}}\left(-\Delta_{\Lambda_{L}^{n}}\right)+\lambda_{0}\right)^{q}\left\langle\phi_{\underline{j}},\left(-\Delta_{\Lambda_{L}^{n}}+\lambda_{0}\right)^{-1} \phi_{\underline{j}}\right\rangle\right)^{2 q} \\
& =2^{2 q} \sum_{\underline{j} \in \mathbb{N} n d}\left(\mu_{\underline{j}}\left(-\Delta_{\Lambda_{L}^{n}}\right)+\lambda_{0}\right)^{-q} \leq C L^{n d} .
\end{aligned}
$$

The last estimate is a direct computation using the explicit form of the Dirichlet eigenvalues.

By [6, Lemma 2.2], we know that, for $q \in \mathbb{N}$ such that $2 q>n d$, there exists $C_{q}>0$ such that, for any measurable subset $\Lambda^{\prime} \subseteq \Lambda_{L^{\prime}}^{n}$ one has

$$
\left\|\left(-\Delta_{\Lambda_{L}^{n}}+\lambda_{0}\right)^{-1 / 2} \mathbf{1}_{\Lambda^{\prime}}\right\|_{\tau_{2 q}}^{2 q} \leq C_{q} \mu\left(\Lambda^{\prime}\right)
$$

Choosing $\Lambda^{\prime}=\left\{\left|V_{i}^{n}\right|>\varepsilon\right\} \cap \Lambda_{L}^{n}$ and taking (2.19) into account, then by combining estimates (2.20)-(2.27), we get that there exists $c$, depending only on $q$ (and the bound in assumption (H.3)), such that

$$
\begin{aligned}
& \text { Trace }\left|\left(H_{L}^{n}+\lambda_{0}\right)^{-q}\left(H_{L}^{n}-z\right)^{-1}-\left(H_{0, L}^{n}+\lambda_{0}\right)^{-q}\left(H_{0, L}^{n}-z\right)^{-1}\right| \\
& \leq c\left(\frac{\varepsilon}{|\Im z z|^{2}} L^{n d}+\frac{1}{|\Im z z|^{2}} L^{n d-(d / 2 q)}+\frac{\varepsilon}{|\Im z|} L^{n d}+\frac{1}{|\Im z|} L^{n d-(d / 2 q)}\right) .
\end{aligned}
$$

By using this inequality in (2.15), we get (2.6) as $\tilde{\varphi}$ being almost analytic, $\bar{\partial} \tilde{\varphi}(z)$ vanishes to any order in $\Im z$ as $z$ approaches the real line. Thus, we completed the proof of Theorem 2.2.

\section{Application to the Interacting Multiparticle Anderson Model}

In the interacting multiparticle Anderson model, we consider a random external potential, that is, $V^{1}=V^{1}(\omega)$. The one particle Anderson potential is of the form

$$
V^{1}(\omega, x)=\sum_{j \in \mathbb{Z}^{d}} \omega_{j} u(x-j),
$$


with a family $\omega_{j}: \Omega \rightarrow \mathbb{R}$ of random variables on $(\Omega, \mathbb{P})$. This one-particle models leads us to the $n$-particle random "background" potential

$$
V^{n}\left(\omega, x_{1}, \ldots, x_{n}\right)=\sum_{k=1}^{n} V^{1}\left(\omega, x_{k}\right)
$$

and the interacting $n$-particle Hamiltonian reads as

$$
H^{n}(\omega)=-\Delta+V_{i}^{n}+V^{n}(\omega)
$$

For the Anderson model, it is known under rather general assumptions that, for a given energy, the normalized counting function defined in assumption (H.1.b) converges almost surely (see, e.g., $[5,9])$. The limit is a nondecreasing function of $E$. Its discontinuity set is countable. By $[9, \mathrm{pp} .311 \mathrm{f}]$, for almost every $\omega$, except at this set, the normalized counting function defined in assumption (H.1.b) then converges. On this set of full measure, we can now apply the results of the last section and get a $\mathbb{P}$-almost sure integrated density of states $N_{\mathrm{ni}}=N$ for both, the noninteracting and interacting $n$-particle system. Note that only translations along a "diagonal" vector $(j, j, \ldots, j) \in \mathbb{Z}^{\text {nd }}$ leave $H^{n}(\omega)$ invariant. Hence, for an application of ergodic theorems (as in the one particle case) for the proof of existence and $\mathbb{P}$-almost sure constancy of $N$, there are typically too few ergodic transformations.

One of the interesting properties of the integrated density of states is its regularity; it is well known to play an important role in the theory of localization for random one-particle models (see, e.g., [10]). Usually, it comes into play through a Wegner estimate, that is, an estimate of the type

$$
\mathbb{E}\left(\operatorname{Trace} \mathbf{1}_{] E_{0}, E_{0}+\eta\right]}\left(H_{\Lambda}^{n}\right)\right) \leq C_{W} \eta|\Lambda|
$$

On the other hand, Corollary 2.3 directly relates the regularity of the IDS of the interacting system to that of the IDS of the single particle Hamiltonian. The regularity of the IDS of the single particle has been the subject of a lot of interest recently (see, e.g., [11, 12]).

We now prove a Wegner estimate; for convenience, we assume the following.

(H.A.2) The single-site potential $u$ is nonnegative, compactly supported, $\|u\|_{L^{\infty}} \leq 1$ and that there is some $c>0$, such that $u(x) \geq c$ for $x \in[-(1 / 2), 1 / 2]^{d}$.

For the proof of a Wegner estimate in the interacting n-particle Anderson model, we can choose rather general probabilistic hypothesis like in [13]:

(H.A.3) $\left(\omega_{j}: \Omega \rightarrow \mathbb{R}\right)_{j \in \mathbb{Z}^{d}}$ is a family of bounded random variables on the probability space $(\Omega, \mathbb{P})$.

When $\mu_{j}$ denotes the conditional probability measure for $\omega_{j}$ at site $j \in \mathbb{Z}^{d}$ conditioned on all the other random variables $\left(\omega_{i}\right)_{i \neq j}$, that is, for all $A \in \mathbb{B}(\mathbb{R})$,

$$
\mu_{j}(A)=\mathbb{P}\left(\left\{\omega_{j} \in A \mid\left(\omega_{i}\right)_{i \neq j}\right\}\right),
$$


then, a Wegner estimate à la [13] uses the quantity

$$
s(\eta):=\sup _{j \in \mathbb{Z}^{d}} \mathbb{E}\left\{\sup _{E \in \mathbb{R}} \mu_{j}([E, E+\eta])\right\}
$$

and is stated as follows.

Theorem 3.1. Let us assume (H.A.2) and (H.A.3), and let $\Lambda \subseteq \mathbb{R}^{\text {nd }}$ be a bounded open cube of side length $\geq 1$, let $H_{\Lambda}^{n}(\omega)$ be the restriction of $H^{n}(\omega)$ to $\Lambda$ with Dirichlet boundary conditions. Then, there exists an increasing function

$$
\begin{gathered}
C_{W}: \mathbb{R} \longrightarrow[0, \infty[ \\
E_{0} \mapsto C_{W}\left(E_{0}\right),
\end{gathered}
$$

such that for all $\eta>0$

$$
\mathbb{E}\left(\operatorname{Trace} \mathbf{1}_{] E_{0}, E_{0}+\eta\right]}\left(H_{\Lambda}^{n}\right)\right) \leq C_{W}\left(E_{0}\right) s(\eta)|\Lambda| .
$$

In order to prove Theorem 3.1, we prove two preparatory lemmas.

Lemma 3.2. Let $\Lambda \subseteq \mathbb{R}^{\text {nd }}$ be an open bounded cube, then the restrictions $H_{i, \Lambda}^{n}$ and $H_{i, \Lambda, N}^{n}$ of $H_{i}^{n}=$ $-\Delta+V_{i}^{n}$ to $\Lambda$ with Dirichlet or Neumann boundary conditions define self-adjoint operators with compact resolvent.

Proof. $V_{i}^{n}$ is infinitesimally $-\Delta$ form bounded according to [4, Theorem X.18], so the infinitesimal form bound

$$
\left|\left\langle\Psi, V_{i}^{n} \Psi\right\rangle\right| \leq \varepsilon\|\nabla \Psi\|^{2}+b_{\varepsilon}\|\Psi\|^{2}
$$

is true for $\Psi \in H^{1}\left(\mathbb{R}^{n d}\right)$, in particular (3.9) is true for $\Psi \in \mathscr{\Phi}\left(-\Delta_{\Lambda}\right)=H_{0}^{1}(\Lambda) \subseteq H^{1}\left(\mathbb{R}^{n d}\right)$. Hence, the form sum defines via representation theorem a self-adjoint operator $H_{i, \Lambda}^{n}=-\Delta_{\Lambda}+\left.V_{i}^{n}\right|_{\Lambda}$. The eigenvalues $\mu_{k}\left(-\Delta_{\Lambda}\right)$ tend to infinity, so by the minimax principle and (3.9), we see that $H_{i, \Lambda}^{n}$ has compact resolvent. The proof of

$$
\left\langle\Psi, V_{i}^{n} \Psi\right\rangle \leq \varepsilon\|\nabla \Psi\|^{2}+c_{\varepsilon}\|\Psi\|^{2}, \quad \Psi \in H^{1}(\Lambda)
$$

uses the extension operator $E_{\Lambda^{\prime}}: H^{1}(\Lambda) \rightarrow H_{0}^{1}\left(\Lambda^{\prime}\right)$ to $\Lambda^{\prime}:=\left\{x \in \mathbb{R}^{n d}: \operatorname{dist}(x, \Lambda)<1\right\}$, which has the properties $\left.E_{\Lambda^{\prime}} \Psi\right|_{\Lambda}=\Psi,\left\|E_{\Lambda^{\prime}} \Psi\right\|_{H^{1}} \leq c_{1}\|\Psi\|_{H^{1}}$ and $\left\|E_{\Lambda^{\prime}} \Psi\right\|_{L^{2}} \leq c_{2}\|\Psi\|_{L^{2}}$; see [14, Satz 5.6 and Folgerung 5.2]. For $E_{\Lambda^{\prime}} \Psi \in H_{0}^{1}\left(\Lambda^{\prime}\right) \subseteq H^{1}\left(\mathbb{R}^{n d}\right)$, we use (3.9), hence by $V_{i}^{n} \geq 0$ and the above properties of $E_{\Lambda^{\prime}}$ we get for $\Psi \in H^{1}(\Lambda)$,

$$
\begin{aligned}
0 & \leq\left\langle\Psi, V_{i}^{n} \Psi\right\rangle \leq\left\langle E_{\Lambda^{\prime}} \Psi, V_{i}^{n} E_{\Lambda^{\prime}} \Psi\right\rangle \leq \varepsilon\left\|\nabla\left(E_{\Lambda^{\prime}} \Psi\right)\right\|_{L^{2}}^{2}+b_{\varepsilon}\left\|E_{\Lambda^{\prime}} \Psi\right\|_{L^{2}}^{2} \\
& =\varepsilon\left\|\left(E_{\Lambda^{\prime}} \Psi\right)\right\|_{H^{1}}^{2}+\left(b_{\varepsilon}-\varepsilon\right)\left\|E_{\Lambda^{\prime}} \Psi\right\|_{L^{2}}^{2} \leq \varepsilon c_{1}^{2}\|\nabla \Psi\|^{2}+\left(c_{2}^{2}\left(b_{\varepsilon}-\varepsilon\right)+\varepsilon c_{1}^{2}\right)\|\Psi\|^{2},
\end{aligned}
$$


which is (3.10). With (3.10) at hand, the proof for Neumann boundary conditions is similar to the Dirichlet case.

Lemma 3.3. Let one assumes (H.A.2) and (H.A.3), and let $\Lambda \subseteq \mathbb{R}^{\text {nd }}$ be a bounded open cube, $\mathbf{j}=$ $\left(j_{1}, \ldots, j_{n}\right) \in \mathbb{Z}^{\text {nd }}$ with $\Lambda_{\mathbf{j}}:=\Lambda \cap \Lambda(\mathbf{j}, 1) \neq \varnothing$ (here, $\left.\Lambda(\mathbf{j}, 1)=\left\{\left|x-j_{k}\right| \leq 1 / 2,1 \leq k \leq n\right\}\right)$, then for every $f \in L^{2}\left(\Lambda_{\mathbf{j}}\right)$,

$$
\mathbb{E}\left\{\left\langle f, \mathbf{1}_{] E_{0}, E_{0}+\eta\right]}\left(H_{\Lambda}^{n}\right) f\right\rangle\right\} \leq \frac{8}{c^{2}} s(\eta)\|f\|^{2} .
$$

Proof. For every $j \in \mathbb{Z}^{d}$, we define $u_{j}: \mathbb{R}^{n d} \rightarrow \mathbb{R}$ by

$$
u_{j}\left(x_{1}, \ldots, x_{n}\right):=\sum_{k=1}^{n} u\left(x_{k}-j\right)
$$

and set $\tilde{\omega}_{j}=\left(\omega_{l}\right)_{l \neq j}$. Fix a component of $\mathbf{j}$, say $j_{1}$, then we get a decomposition

$$
V^{n}\left(\omega, x_{1}, \ldots, x_{n}\right)=\omega_{j_{1}} u_{j_{1}}\left(x_{1}, \ldots, x_{n}\right)+\sum_{\substack{l \in \mathbb{Z}^{d} \\ l \neq j_{1}}} \omega_{l} u_{l}\left(x_{1}, \ldots, x_{n}\right)
$$

of the random potential $V^{n}(\omega)$, and the same is true for $H_{\Lambda}^{n}(\omega)$ :

$$
H_{\Lambda}^{n}(\omega)=-\Delta_{\Lambda}+\sum_{\substack{l \in \mathbb{Z}^{d} \\ l \neq j_{1}}} \omega_{l} u_{l} \mathbf{1}_{\Lambda}+\omega_{j_{1}} u_{j_{1}} \mathbf{1}_{\Lambda}=: \widetilde{H}_{\Lambda}^{n}\left(\widetilde{\omega}_{j_{1}}\right)+\omega_{j_{1}} u_{j_{1}} \mathbf{1}_{\Lambda}
$$

By the covering condition $u \mathbf{1}_{[-1 / 2,1 / 2]^{d}} \geq c$ on the single site-potential $u$, we get $u_{j_{1}} \geq c \mathbf{1}_{\Lambda_{\mathrm{j}}}$, hence we can write $f=g u_{j_{1}}$, where $g(x)=f(x) / u_{j_{1}}(x)$ almost everywhere, so $\|g\| \leq c^{-1}\|f\|$. By spectral calculus,

$$
\int_{E_{0}}^{E_{0}+\eta} d E\left\langle\varphi, \Im(H-E-i \eta)^{-1} \varphi\right\rangle \geq \frac{\pi}{4}\left\langle\varphi, \mathbf{1}_{] E_{0}, E_{0}+\eta\right]}(H) \varphi\right\rangle,
$$

for every self-adjoint $H$, see [13], (3.9). The equalities and estimates in (3.15) and (3.16) allow us to put the problem into a form, where the results of spectral averaging, [11, Section 3 ], apply

$$
\begin{aligned}
& \mathbb{E}\left\langle f, \mathbf{1}_{] E_{0}, E_{0}+\eta\right]}\left(H_{\Lambda}^{n}\right) f\right\rangle=\mathbb{E} \int_{\mathbb{R}} d \mu_{j_{1}}\left(\omega_{j_{1}}\right)\left\langle g, u_{j_{1}} \mathbf{1}_{] E_{0}, E_{0}+\eta\right]}\left(\widetilde{H}_{\Lambda}^{n}\left(\tilde{\omega}_{j_{1}}\right)+\omega_{j_{1}} u_{j_{1}}\right) u_{j_{1}} g\right\rangle \\
& \leq \frac{4}{\pi} \mathbb{E} \int_{\mathbb{R}} d \mu_{j_{1}}\left(\omega_{j_{1}}\right) \int_{E_{0}}^{E_{0}+\eta} d E \Im\left\langle g, u_{j_{1}}\left(\widetilde{H}_{\Lambda}^{n}(\tilde{\omega})+\omega_{j_{1}} u_{j_{1}}-E-i \eta\right)^{-1} u_{j_{1}} g\right\rangle \\
& \leq \frac{8}{c^{2}}\|f\|^{2} s(\eta)
\end{aligned}
$$


Proof of Theorem 3.1. By (H.A.2) and (H.A.3), we get a $\mathbb{P}$-almost sure bound $\left\|V^{n}(\omega)\right\| \leq\left|V^{n}\right|$, then Lemma 3.2 implies that the restrictions $H_{\Lambda}^{n}(\omega)$ and $H_{\Lambda, N}^{n}(\omega)$ of $H^{n}(\omega)$ to a bounded open cube with Dirichlet or Neumann boundary conditions define self-adjoint operators with compact resolvent $\mathbb{P}$-almost sure. Let $J:=\left\{\mathbf{j} \in \mathbb{Z}^{\text {nd }}: \Lambda(\mathbf{j}, 1) \cap \Lambda \neq \varnothing\right\}$ and for $\mathbf{j} \in J$ set $\Lambda_{\mathbf{j}}:=$ $\Lambda(\mathbf{j}, 1) \cap \Lambda$. Then $\Lambda^{\prime}:=\Lambda \backslash \cup_{\mathbf{j} \in J} \Lambda_{\mathbf{j}}$ has Lebesgue measure 0 , so by [15, XIII.15, Propositions 3 and 4$]$, we have

$$
-\Delta_{\Lambda} \geq-\Delta_{\Lambda, N} \geq-\Delta_{\Lambda \backslash \Lambda^{\prime}, N}=\bigoplus_{\mathbf{j} \in J}\left(-\Delta_{\Lambda_{\mathrm{j}}, N}\right)
$$

So with $H_{i, \Lambda_{\mathrm{j}}, N}^{n}$ defined in Lemma 3.2, we get $\mathbb{P}$-almost sure:

$$
H_{\Lambda}^{n}(\omega) \geq H_{\Lambda, N}^{n}:=\bigoplus_{\mathbf{j} \in J} H_{i, \Lambda_{\mathrm{j}}, N}^{n}-\left|V^{n}\right|
$$

By spectral calculus,

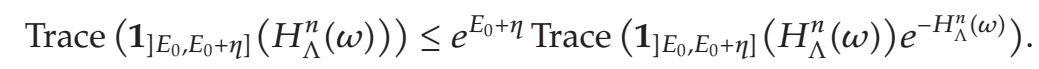

Let $\left(\varphi_{k}(\omega)\right)_{k \in \mathbb{N}}$ be the orthogonal basis of $L^{2}(\Lambda)$ consisting out of eigenvectors of $H_{\Lambda}^{n}(\omega)$ to eigenvalues $\mu_{k}(\omega)$ and let $\left.\left.M(\omega):=\left\{k \in \mathbb{N}: \mu_{k}(\omega) \in\right] E_{0}, E_{0}+\eta\right]\right\}$, then

$$
\begin{aligned}
\operatorname{Trace}\left(\mathbf{1}_{] E_{0}, E_{0}+\eta\right]}\left(H_{\Lambda}^{n}(\omega)\right) e^{-H_{\Lambda}^{n}(\omega)}\right) & =\sum_{k \in M(\omega)} e^{-\left\langle\varphi_{k}(\omega), H_{\Lambda}^{n}(\omega) \varphi_{k}(\omega)\right\rangle} \\
& \leq \sum_{k \in M(\omega)} e^{-\left\langle\varphi_{k}(\omega), H_{\Lambda, N}^{n} \varphi_{k}(\omega)\right\rangle} \\
& \leq \sum_{k \in M(\omega)}\left\langle\varphi_{k}(\omega), e^{-H_{\Lambda, N}^{n}} \varphi_{k}(\omega)\right\rangle \\
& =\operatorname{Trace}\left(\mathbf{1}_{] E_{0}, E_{0}+\eta\right]}\left(H_{\Lambda}^{n}(\omega)\right) e^{-H_{\Lambda, N}^{n}}\right)
\end{aligned}
$$

where the last estimate follows from Jensen's inequality. Let $\left(\phi_{k, \mathrm{j}}\right)_{k \in \mathbb{N}}$ be an orthonormal basis of $L^{2}\left(\Lambda_{\mathbf{j}}\right)$ consisting of eigenvectors of $H_{i, \Lambda_{j}, N}^{n}$ to the eigenvalues $E_{k, j}$, then

$$
\operatorname{Trace}\left(\mathbf{1}_{] E_{0}, E_{0}+\eta\right]}\left(H_{\Lambda}^{n}(\omega)\right) e^{-H_{\Lambda, N}^{n}}\right)=\sum_{k \in \mathbb{N}} \sum_{\mathbf{j} \in J}\left\langle\phi_{k, \mathbf{j}}, \mathbf{1}_{] E_{0}, E_{0}+\eta\right]}\left(H_{\Lambda}^{n}(\omega)\right) \phi_{k, \mathbf{j}}\right\rangle e^{-E_{k, j}+\left|V^{n}\right|}
$$

As $\phi_{k, j} \in L^{2}\left(\Lambda_{\mathbf{j}}\right)$ and $\left\|\phi_{k, j}\right\| \leq 1$, Lemma 3.3 implies

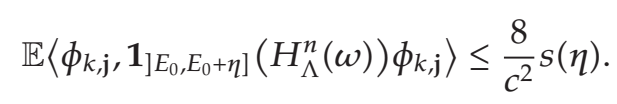


As $V_{i}^{n}$ is nonnegative, the eigenvalues $E_{k, \mathrm{j}}$ of $H_{i, \Lambda_{\mathrm{j}}, N}^{n}=-\Delta_{\Lambda_{\mathrm{j}}, N}+\left.V_{i}^{n}\right|_{\Lambda_{\mathrm{j}}}$ are estimated from what follows by the eigenvalues of $-\Delta_{\Lambda_{\mathrm{j}}, N}$. These are known explicitly, see [15, page 266], which can be used to estimate

$$
\sum_{k \in \mathbb{N}} \sum_{\mathbf{j} \in J} e^{-E_{k, j}} \leq \operatorname{Card}(J)\left(\frac{e^{\pi^{2}}}{e^{\pi^{2}}-1}\right)^{n d} .
$$

If the side-length of $\Lambda$ is bigger than 1 , then $\operatorname{Card}(J) \leq 3^{\text {nd }}|\Lambda|$, so when applying expectation value to the chain of inequalities (3.20) to (3.24), it implies

$$
\mathbb{E}\left(\text { Trace } \mathbf{1}_{] E_{0}, E_{0}+\eta\right]}\left(H_{\Lambda}^{n}\right)\right) \leq e^{E_{0}+\eta+\left|V^{n}\right|}\left(\frac{3 e^{\pi^{2}}}{e^{\pi^{2}}-1}\right)^{n d} \frac{8}{c^{2}} s(\eta)|\Lambda|
$$

Under the assumptions (H.A.2) and (H.A.3), we have

$$
N(E)=\mathbb{E}\left(N(E, \cdot) \mathbf{1}_{\Omega^{\prime}}\right)=\mathbb{E}(N(E, \cdot)),
$$

hence by the Wegner estimate we can deduce regularity properties of $N$ from those of the conditioned measures $\left(\mu_{j}\right)_{j \in \mathbb{Z}^{d}}$ via

$$
0 \leq N(E+\eta)-N(E) \leq C_{W}(E+\eta) s(\eta)
$$

\section{References}

[1] W. Kirsch, "A Wegner estimate for multi-particle random Hamiltonians," Zhurnal Matematicheskŏ Fiziki, Analiza, Geometrii, vol. 4, no. 1, pp. 121-127, 2008.

[2] V. Chulaevsky, "Wegner-Stollmann type estimates for some quantum lattice systems," in Adventures in Mathematical Physics, vol. 447 of Contemporary Mathematics, pp. 17-28, American Mathematical Society, Providence, RI, USA, 2007.

[3] M. Aizenman and S. Warzel, "Localization bounds for multiparticle systems," preprint, 2008, http://arxiv.org/abs/0809.3436.

[4] M. Reed and B. Simon, Methods of Modern Mathematical Physics. II: Fourier Analysis, Self-Adjointness, Academic Press, New York, NY USA, 1975.

[5] L. Pastur and A. Figotin, Spectra of Random and Almost-Periodic Operators, vol. 297 of Grundlehren der Mathematischen Wissenschaften, Springer, Berlin, Germany, 1992.

[6] F. Klopp and L. Pastur, "Lifshitz tails for random Schrödinger operators with negative singular Poisson potential," Communications in Mathematical Physics, vol. 206, no. 1, pp. 57-103, 1999.

[7] F. Klopp, "Internal Lifshits tails for random perturbations of periodic Schrödinger operators," Duke Mathematical Journal, vol. 98, no. 2, pp. 335-396, 1999.

[8] B. Simon, Trace Ideals and Their Applications, vol. 120 of Mathematical Surveys and Monographs, American Mathematical Society, Providence, RI, USA, 2nd edition, 2005.

[9] R. Carmona and J. Lacroix, Spectral Theory of Random Schrödinger Operators, Probability and Its Applications, Birkhäuser, Boston, Mass, USA, 1990.

[10] P. Stollmann, Caught by Disorder: Bound States in Random Media, vol. 20 of Progress in Mathematical Physics, Birkhäuser, Boston, Mass, USA, 2001.

[11] J.-M. Combes, P. D. Hislop, and F. Klopp, "Local and global continuity of the integrated density of states," in Advances in Differential Equations and Mathematical Physics (Birmingham, AL, 2002), vol. 327 of Contemporary Mathematics, pp. 61-74, American Mathematical Society, Providence, RI, USA, 2003. 
[12] G. Stolz, "Strategies in localization proofs for one-dimensional random Schrödinger operators," Proceedings of the Indian Academy of Sciences. Mathematical Sciences, vol. 112, no. 1, pp. 229-243, 2002.

[13] J.-M. Combes, P. D. Hislop, and F. Klopp, "An optimal Wegner estimate and its application to the global continuity of the integrated density of states for random Schrödinger operators," Duke Mathematical Journal, vol. 140, no. 3, pp. 469-498, 2007.

[14] J. Wloka, Partielle Differentialgleichungen, B. G. Teubner, Stuttgart, Germany, 1982.

[15] M. Reed and B. Simon, Methods of Modern Mathematical Physics. IV: Analysis of Operators, Academic Press, New York, NY, USA, 1978. 


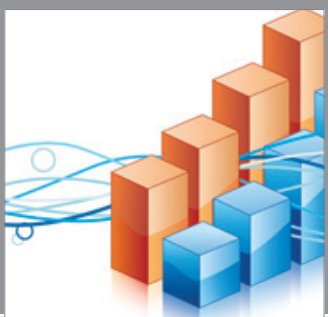

Advances in

Operations Research

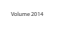

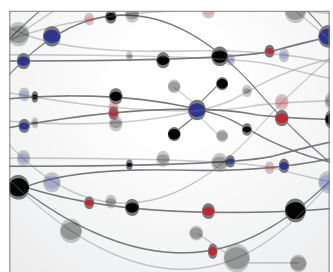

\section{The Scientific} World Journal
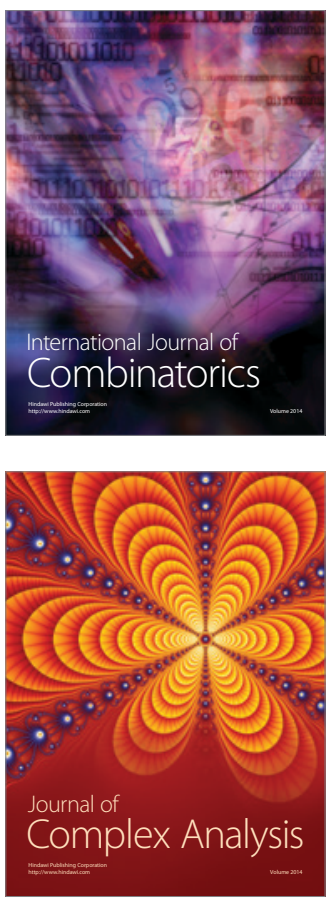

International Journal of

Mathematics and

Mathematical

Sciences
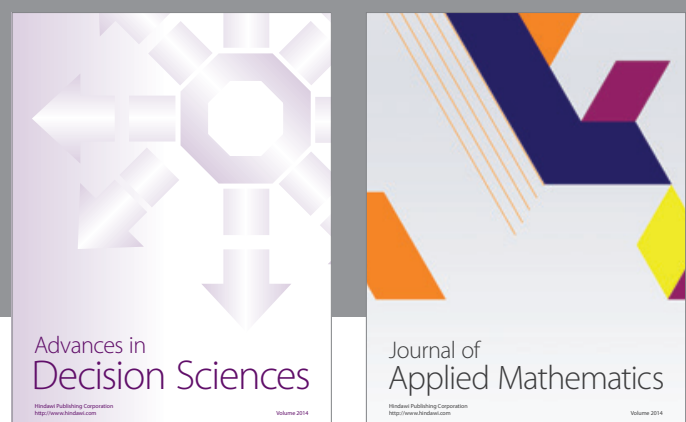

Journal of

Applied Mathematics
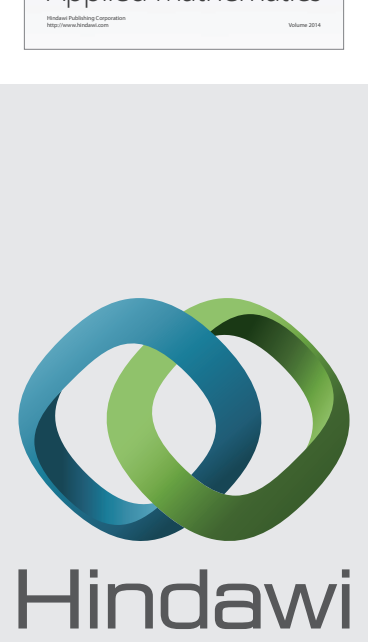

Submit your manuscripts at http://www.hindawi.com
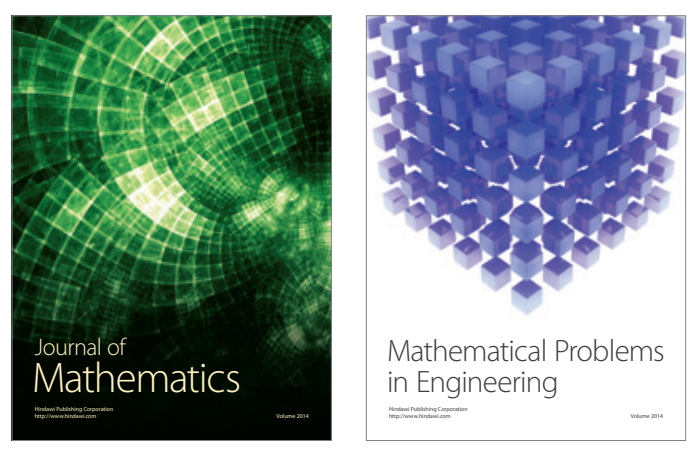

Mathematical Problems in Engineering
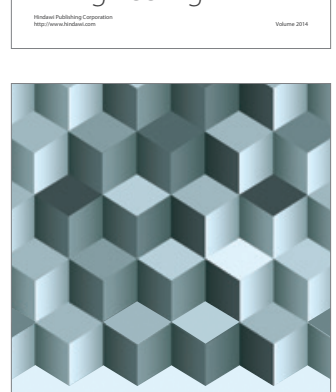

Journal of

Function Spaces
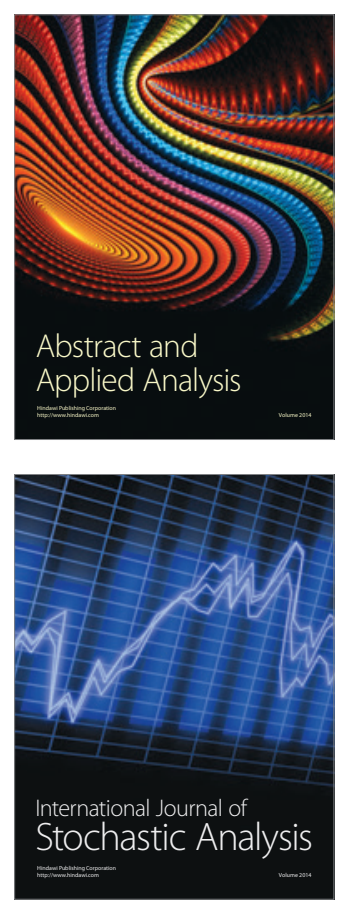

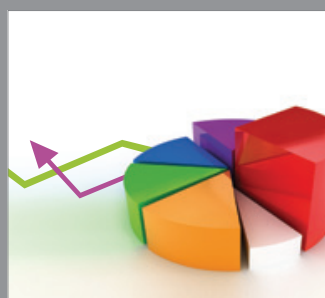

ournal of

Probability and Statistics

Promensencen
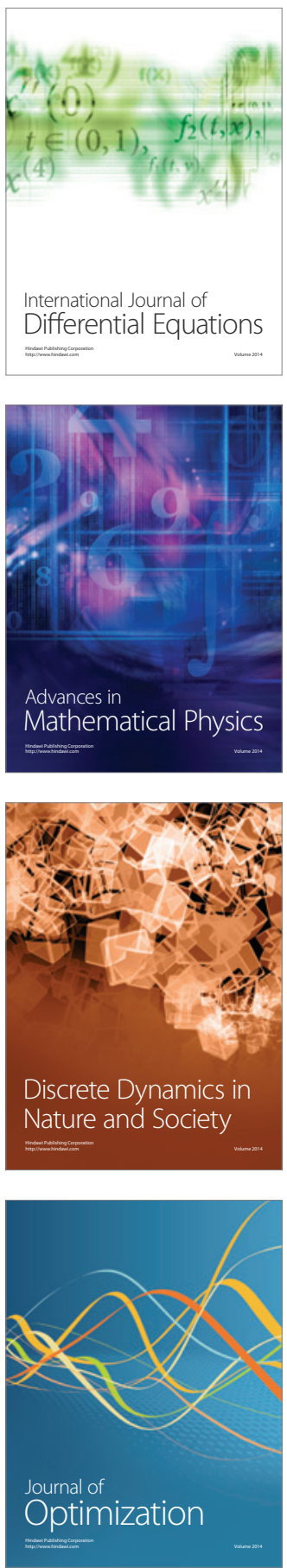